\title{
SCHWERPUNKTTHEMA „DIGITALE UND SOZIALE TRANSFORMATION“
}

Liebe Leser*innen,

sowohl die digitale als auch die damit untrennbar verbundene soziale Transformation zählen zwar schon seit Jahrzehnten zum Alltag vieler Bibliothekar*innen, durch die COVID-19-Pandemie und diverse Digitalisierungsoffensiven wurden aber beide noch einmal massiv beschleunigt.

So lancierte beispielsweise das Bundesministerium für Bildung, Wissenschaft und Forschung (BMBWF) im Jahre 2019 die Ausschreibung "Digitale und soziale Transformation in der Hochschulbildung" (DigiSoz), um profilbildende, strukturentwickelnde und zukunftsweisende Digitalisierungsvorhaben öffentlicher Universitäten zu ermöglichen. Besonderes Augenmerk wurde dabei in inhaltlicher Hinsicht auf die folgenden Bereiche gerichtet: Digitalisierung in der Lehre und im Lernen \& Learning Analytics, Skills für das digitale Zeitalter - Auf dem Weg zum Curriculum 4.0, Digitale Transformation für die soziale Dimension nutzen, Open Science sowie E-Administration - Digitalisierung in der Verwaltung, wobei auch insbesondere der Zusammenschluss zu Kooperationen und Netzwerken, die Strukturveränderung und Systemwirkung sowie die Unterstützung von Change-Management-Prozessen im Vordergrund standen. ${ }^{1}$ Von den insgesamt 71 Projekteinreichungen, die bis 15. September 2019 eingelangt waren, erhielten schließlich im Jänner 202035 einen Zuschlag. ${ }^{2}$ An gut einem Viertel dieser Vorhaben sind u.a. eine, mehrere oder - wie im Falle von Austrian Transition to Open Access 2 (AT2OA ${ }^{2}$ ) - alle Universitätsbibliotheken direkt oder indirekt beteiligt. Gleiches gilt im europäischen Kontext auch hinsichtlich diverser Initiativen, die im Zusammenhang mit der Etablierung der European Open Science Cloud (EOSC) ${ }^{3}$ gestartet wurden. ${ }^{4}$

Die vorliegende Ausgabe gibt einen Einblick in die Ziele, Tätigkeiten und (erste) Ergebnisse zahlreicher dieser Projekte mit Bibliotheksbeteiligung. ${ }^{5}$

\section{Schwerpunktthema}

Mit der Gründung des Forums Digitalisierung (FoDi) der österreichischen Universitätenkonferenz (uniko), dem alle für den Bereich Digitalisierung 
zuständigen Vizerektor*innen der öffentlichen Universitäten angehören, wurde Anfang März 2020 eine Plattform ins Leben gerufen, deren Hauptaugenmerk auf der strategischen Entwicklung des digitalen österreichischen Hochschulraums liegt. In ihrem Beitrag „Digitale Transformation und Innovation für Universitäten" heben die beiden Vorsitzenden des FoDis Ronald Maier und Claudia von der Linden u.a. hervor, wie wichtig es ist, Bewusstsein für Potenziale und Risiken der Digitalisierung zu entwickeln sowie die Reichweite und Folgen des digitalen Wandels beurteilen zu können und diesen aktiv mitzugestalten, und schildern darüber hinaus, wie die universitätsübergreifende Zusammenarbeit genau funktioniert und welche Rolle den Universitätsbibliotheken hinsichtlich des Themenkomplexes Open Science zukommt. ${ }^{6}$

Einen Überblick über die Ziele und aktuellen Ergebnisse von FAIR Data Austria geben Susanne Blumesberger, Raman Ganguly, Nikos Gänsdorfer, Eva Gergely, Alexander Gruber, Ilire Hasani-Mavriqi, Tereza Kalová, Christoph Ladurner, Therese Macher, Tomasz Miksa, Barbara Sánchez Solís, Hermann Schranzhofer, Christiane Stork, Sarah Stryeck und Heike Töricht in ihrem Beitrag „FAIR Data Austria - Aligning the Implementation of FAIR Tools and Services". ${ }^{7}$ Im Rahmen des Projekts werden sowohl Tools für das Management des Lebenszyklus von Forschungsdaten (einschließlich maschinenverarbeitbarer Datenmanagementpläne, kurz maDMPs) und Repositorien für die Langzeitarchivierung von Forschungsergebnissen sowie RDM-Schulungen und -Support-Services, als auch Modelle und Profile für Data Stewards entwickelt und etabliert sowie das FAIR Office Austria ${ }^{8}$ eingerichtet.

Gürsoy Doğtaş, Marc-Paul Ibitz, Astrid Poyer und Charlotte Reuß erläutern in ihrem Beitrag „IMAGE+ Platform for Open Art Education. Strategien zu einer unabhängigen Infrastruktur für Forschung \& Lehre"9 die ersten Schritte zur Etablierung einer auf Open Source-Software basierenden Bild- und Bildforschungsplattform, die ihren Nutzer*innen einen umfassenden Bestand an hochwertigen, mit Metadaten angereicherten digitalen Bildreproduktionen künstlerischer Arbeiten bietet. Neben der transparenten Vermittlung von Bildrechten liegt der Fokus dabei insbesondere auf der diskriminierungskritischen Überprüfung der Bilder und ihrer Metadaten sowie der Erarbeitung und Anwendung antirassistischer und dekolonialer Praktiken bei der Erschließung der Inhalte.

Auch bei iMooX.at ${ }^{10}$ handelt es sich um eine dem Open Education-Gedanken folgende elektronische Infrastruktur. Die bereits 2013 von der Universität Graz und der Technischen Universität Graz gegründete MOOCPlattform bietet ihren Nutzer*innen kostenlose, offen lizenzierte Online- 
Kurse an und soll zukünftig allen österreichischen Hochschulen als Service zur Verfügung stehen. In seinem Beitrag „iMooX - eine MOOC-Plattform für alle (Hochschulen)“"11 skizziert Martin Ebner ihre Entstehungsgeschichte und wesentliche Meilensteine sowie die noch geplanten Schritte zur weiteren Entwicklung der Plattform.

Der nachhaltige Umgang mit und die Erhöhung der Sichtbarkeit von Forschungsinformationen sowie die Entlastung des Wissenschaftsbetriebs sind die wesentlichen Anliegen des Projekts RIS Synergy. ${ }^{12}$ Madeleine Harbich, Ulrike Hicker und Sabine Neff beschreiben in ihrem Beitrag „RIS Synergy: Standards schaffen - Services digitalisieren - Expertise vernetzen“, ${ }^{13}$ wie diese Ziele mit Hilfe von Schnittstellen und Standards für Systeme von Fördergebern, Forschungsstätten und der öffentlichen Verwaltung sowie der Eruierung der Rahmenbedingungen und Anforderungen für die Etablierung eines international sichtbaren und vernetzten Forschungsportals erreicht werden sollen.

Nach dem offiziellen Startschuss für die European Open Science Cloud (EOSC) und der Verabschiedung der „Vienna Declaration on the European Open Science Cloud“14 am 23. November 2018 in Wien wurde am 15. Oktober 2021 ein weiterer wichtiger Meilenstein auf österreichischer Ebende in dieser Hinsicht erreicht. ${ }^{15}$ Der Beitrag „The Austrian EOSC Mandated Organisation / The EOSC Support Office Austria"16 von Susanne Blumesberger, Florian Brandt, Paolo Budroni, Juliana De Mello Castro Giroletti, Andreas Ferus, Katharina Flicker, Raman Ganguly, Beate Guba, Stefan Hanslik, Ilire Hasani-Mavriqi, Lisa Hönegger, Tereza Kalová, Michael Kranewitter, Bernd Logar, Christian Panigl, Heimo Rainer, Andreas Rauber, Barbara Sánchez Solís, Bernd Saurugger, Chris Schubert, Katrin Vohland und Kerstin Zimmermann bietet einen Einblick in einige Überlegungen hinsichtlich der Umsetzung der European Open Science Cloud (EOSC) auf nationaler Ebene und schildert die Entstehung und Organisationstruktur der österreichischen EOSC Mandated Organisation und des EOSC Support Office Austria.

Der Grundstein zum Aufbau der EOSC wurde allerdings bereits im Rahmen verschiedener Horizon 2020-Projekte, wie bspw. EOSC-Pillar ${ }^{17}$ und seiner drei „Schwesterprojekte“, der 5b-Projekte, gelegt. In ihrem Beitrag "Reflections on the regional approach for Open Science in Europe - EOSC-Pillar and the other " $5 \mathrm{~b}$ projects" 18 berichten Paolo Budroni und Lisa Hönegger nicht nur über die vielfältigen Aktivitäten, die zum Zwecke des Aufbaus der EOSC durchgeführt wurden, sondern zeigen auch explizit auf, wie wichtig es ist, bei einer nationalen „Verankerung“ der EOSC auf individuelle, regionale Bedürfnisse einzugehen. 


\section{Beiträge}

Dass die digitale Transformation Bibliothekar*innen bereits seit Jahrzehnten beschäftigt, zeigen Karin Lackner und Lisa Schilhan in ihrem Beitrag „Der Einzug der EDV im österreichischen Bibliothekswesen am Beispiel der Universitätsbibliothek Graz" ${ }^{19}$ wunderbar auf, kam es doch dort durch den Einsatz von EDV-Systemen ab den 1970er Jahren zu einem radikalen Wandel in der Benutzung und Verwaltung der UB. Ihr Artikel liefert einen historischen Überblick über die Anfänge, die Entwicklung und Verbreitung der elektronischen Bibliothekssysteme im Allgemeinen sowie an der Universitätsbibliothek Graz im Speziellen. Vorgestellt werden die im Lauf der Jahrzehnte an der UB Graz eingesetzten Bibliothekssysteme GRIBS, EMILE, FBInfo, BIBOS, ALEPH und ALMA sowie die Entwicklung von den ersten Online- über die CD-ROM-Datenbanken bis hin zum modernen Datenbank-Retrieval.

\section{Reports}

Auch die Bedeutung der exemplarspezifischen Erschließung - also der Darstellung von besonderen Merkmalen und der Vorgeschichte eines bestimmten Buches im Katalog - ist in den vergangenen Jahren im Zusammenhang mit der Erforschung und Rekonstruktion alter und wertvoller Bestände, der NS-Provenienzforschung sowie der Digitalisierung an sich gewachsen. Um gemeinsame Lösungen für die Erschließung, vor allem aber für die verbundweite Darstellung und Suchbarkeit von Exemplarspezifika zu finden, wurde eine Arbeitsgruppe der Zentralen Redaktion des Österreichischen Bibliothekenverbundes eingerichtet. In ihrem Beitrag „Exemplarspezifische Erschließung im Österreichischen Bibliothekenverbund “20 präsentieren Stefan Alker-Windbichler, Karl-Heinz Bauer, Wolfgang Bruckner, Nadine Cerny, Monika Kiegler-Griensteidl und Josef Labner die Ergebnisse der Arbeitsgruppe, die Arbeitsunterlagen sowohl für die Erschließung, das Setup der Verbundsuchmaschine, das spezifische Suchen, exemplarspezifische Links als auch die provisorische Nutzung von Normdaten umfassen.

Wir wünschen Ihnen eine interessante und anregende Lektüre und freuen uns über Ihr Feedback.

Ihr Editorial Board der Mitteilungen der VÖB 
1 Bundesministerium für Bildung, Wissenschaft und Forschung, Ausschreibungen, Digitalisierung/SozDim 2019: https://unicontrolling.bmbwf. gv.at/index.php?option=com_content\&view=category\&id=20\&ltem id $=101$

2 Bundesministerium für Bildung, Wissenschaft und Forschung (2020). Digitale und soziale Transformation: Ausgewählte Digitalisierungsvorhaben an öffentlichen Universitäten 2020 bis 2024. https://pubshop. bmbwf.gv.at/index.php?rex_media_type=pubshop_download\&rex_ media_file=digital_uni.pdf

3 European Open Science Cloud (EOSC): https://www.eosc.eu/about-eosc

4 Vgl. hierzu auch bspw. Bauer, B. (2018). Präsentation der European Open Science Cloud an der Universität Wien (Wien, 23. November 2018). Mitteilungen der Vereinigung Österreichischer Bibliothekarinnen und Bibliothekare, 71(3-4), 524-529. https://doi.org/10.31263/ voebm.v71i3-4.2171, sowie European Commission (2018). The Vienna Declaration on the European Open Science Cloud. https://eosclaunch.eu/declaration/

5 DerStatus quo aller DigiSoz-Projekte wurde vom Forum Digitalisierung im Herbst 2021 als Postersammlung zusammengefasst: https://uniko.ac.at/ modules/download.php?key=28271_DE_O\&f=1\&jt=7906\&cs=8C07. Zwei weitere Projekte, Austrian Transition to Open Access 2: https:// www.at2oa.at/at2oa2_home.html und Open Education Austria Advanced: https://www.openeducation.at/, werden in einer der kommenden Ausgaben der Mitteilungen der VÖB näher vorgestellt.

6 Maier, R., \& von der Linden, C. (2021). Digitale Transformation und Innovation für Universitäten: Beitrag der Vertreter*innen des Forums Digitalisierung der Österreichischen Universitätenkonferenz (uniko). Mitteilungen der Vereinigung Österreichischer Bibliothekarinnen und Bibliothekare, 74(2). https://doi.org/10.31263/voebm.v74i2.6380

7 Blumesberger, S., Gänsdorfer, N., Ganguly, R., Gergely, E., Gruber, A., Hasani-Mavriqi, I., Kalová, T., Ladurner, C., Macher, T., Miksa, T., Sanchéz Solís, B., Schranzhofer, H., Stork, C., Stryeck, S., \& Thöricht, H. (2021). FAIR Data Austria - Abstimmung der Implementierung von FAIR Tools und Services. Mitteilungen der Vereinigung Österreichischer Bibliothekarinnen und Bibliothekare, 74(2). https://doi.org/10.31263/ voebm.v74i2.6379

8 FAIR Office Austria: https://fair-office.at/

9 Doğtaş, G., Ibitz, M.-P., Poyer, A., \& Reuß, C. (2021). IMAGE+ Platform for Open Art Education: Strategien zu einer unabhängigen Infrastruktur für Forschung \& Lehre. Mitteilungen der Vereinigung Ös- 
terreichischer Bibliothekarinnen und Bibliothekare, 74(2). https://doi. org/10.31263/voebm.v74i2.6397

10 iMooX: https://imoox.at/mooc/

11 Ebner, M. (2021). iMooX - eine MOOC-Plattform für alle (Hochschulen). Mitteilungen der Vereinigung Österreichischer Bibliothekarinnen und Bibliothekare, 74(2). https://doi.org/10.31263/voebm. v74i2.6271

12 RIS Synergy: https://forschungsdaten.at/ris/

13 Harbich, M., Hicker, U., \& Neff, S. (2021). RIS Synergy: Standards schaffen - Services digitalisieren - Expertise vernetzen. Mitteilungen der Vereinigung Österreichischer Bibliothekarinnen und Bibliothekare, 74(2). https://doi.org/10.31263/voebm.v74i2.6269

14 The Vienna Declaration on the European Open Science Cloud. https:// eosc-launch.eu/declaration/. Siehe hierzu bspw. auch Bauer, B. (2018). Präsentation der European Open Science Cloud an der Universität Wien (Wien, 23. November 2018). Mitteilungen der Vereinigung Österreichischer Bibliothekarinnen und Bibliothekare, 71(3-4), 524-529. https://doi.org/10.31263/voebm.v71i3-4.2171

$15 \mathrm{Vgl}$. hierzu auch die offizielle APA-Pressemitteilung „Austrian EOSC Mandated Organisation offiziell gegründet - ein Meilenstein für Open Science". https://www.ots.at/presseaussendung/OTS_20211015_OTS0129/austrian-eosc-mandated-organisation-offiziell-gegruendet-ein-meilensteinfuer-open-science, sowie Flicker, K., Giroletti, J., \& Saurugger, B. (2021). Austrian EOSC Mandated Organisation founded: A milestone for Open Science in Austria. Zenodo. https://doi.org/10.5281/zenodo.5588259

16 Blumesberger, S., Brandt, F., Budroni, P., De Mello Castro Giroletti, J., Ferus, A., Flicker, K., Ganguly, R., Guba, B., Hanslik, S., HasaniMavriqi, I., Hönegger, L., Kalová, T., Kranewitter, M., Logar, B., Panigl, C., Rainer, H., Rauber, A., Sánchez Solís, B., Saurugger, B., Schubert, C., Vohland, K., \& Zimmermann, K. (2021). The Austrian EOSC Mandated Organisation / The EOSC Support Office Austria. Mitteilungen der Vereinigung Österreichischer Bibliothekarinnen und Bibliothekare, 74(2). https://doi.org/10.31263/voebm.v74i2.6270

17 EOSC-Pillar: https://www.eosc-pillar.eu/

18 Budroni, P., \& Hönegger, L. (2021). Reflections on the regional approach for Open Science in Europe - EOSC-Pillar and the other "5b projects". Mitteilungen der Vereinigung Österreichischer Bibliothekarinnen und Bibliothekare, 74(2). https://doi.org/10.31263/voebm.v74i2.6282

19 Lackner, K. \& Schilhan, L. (2021). Der Einzug der EDV im österreichischen Bibliothekswesen am Beispiel der Universitätsbibliothek Graz. 
Mitteilungen der Vereinigung Österreichischer Bibliothekarinnen und Bibliothekare, 74(2). https://doi.org/10.31263/voebm.v74i2.6395 20 Alker-Windbichler, S., Bauer, K.-H., Bruckner, W., Cerny, N., KieglerGriensteidl, M., \& Labner, J. (2021). Exemplarspezifische Erschließung im Österreichischen Bibliothekenverbund: Ergebnisse einer Arbeitsgruppe der Zentralen Redaktion. Mitteilungen der Vereinigung Österreichischer Bibliothekarinnen und Bibliothekare, 74(2). https://doi. org/10.31263/voebm.v74i2.6411

DOI: https://doi.org/10.31263/voebm.v74i2.6556

Dieses Werk ist - exkl. einzelner Logos und Abbildungen - lizenziert unter einer Creative-Commons-Lizenz Namensnennung 4.0 International-Lizenz 